\title{
Altered efficacy of AT1R-targeted treatment after spontaneous cancer cell-AT1R upregulation
}

\author{
Eleanor I Ager*, Shu Wen Wen, Joyna Chan, Way W Chong, Jaclyn H Neo and Christopher Christophi
}

\begin{abstract}
Background: Targeting of the renin angiotensin system (RAS) reduces tumour growth in experimental models of cancer. We aimed to establish if combined targeting of the 'classical' and 'alternative' arms of the RAS could result in synergistic inhibition of colorectal cancer (CRC) liver metastases.

Methods: Immediately following induction of CRC liver metastases through intrasplenic injection of murine CRC cells, treatment with irbesartan (AT1R blocker; $50 \mathrm{mg} / \mathrm{kg} /$ day s.c.), captopril (ACE inhibitor; $750 \mathrm{mg} / \mathrm{kg} /$ day i.p.), CGP42112A (AT2R agonist; $0.6 \mu \mathrm{g} / \mathrm{kg} / \mathrm{hr}$ i.p.), and/or ANG-(1-7) (24 $\mathrm{\mu g} / \mathrm{kg} / \mathrm{hr}$ i.p.) began and continued for 21 days. Liver to body weight ratio and/or stereology were used as a measure of tumour burden. Immunohistochemistry was used to determine AT1R and VEGF expression as well as proliferation (Ki67), apoptosis (active caspase 3) and angiogenesis (CD34).

Results: Combined RAS therapies failed to improve upon single arm therapies. However, while irbesartan previously inhibited tumour growth in this model, in the current experiments irbesartan failed to affect tumour burden. Subsequent analysis showed a cancer-cell specific upregulation of the angiotensin II type I receptor (AT1R) in irbesartan-insensitive compared to irbesartan-sensitive tumours. The upregulation of AT1R was associated with an increase in proliferation and VEGF expression by cancer cells. While animals bearing irbesartan-sensitive tumours showed a marked decrease in the number of proliferating cells in the liver and VEGF-expressing infiltrating cells in the tumour following AT1R treatment, these were unchanged by treatment in animals bearing irbesartaninsensitive (high AT1R expressing) tumours.
\end{abstract}

Conclusions: Although the results do not support increased efficacy of combined treatment, they provide intriguing evidence of the importance of RAS expression in determining patient response and tumour growth potential and suggest that components of the RAS could be used as biomarkers to aid in patient selection.

Keywords: renin angiotensin system, liver metastases, biomarker, combination therapy

\section{Background}

Metastasis to the liver is the leading cause of death in patients with colorectal cancer (CRC)[1]. We previously showed that targeting of the renin angiotensin system (RAS) with either an angiotensin (ANG) II type I receptor (AT1R) blocker (irbesartan) or an angiotensin converting enzyme (ACE) inhibitor (captopril) could inhibit tumour growth in an orthotopic syngeneic mouse model of CRC liver metastases [2,3]. ACE is responsible for converting inactive ANG I into the key active peptide of the classical RAS, ANG II. The AT1R mediates

\footnotetext{
* Correspondence: eager@unimelb.edu.au
The Department of Surgery, Austin Health, The University of Melbourne,

* Correspondence: eager@unimelb.edu.au
The Department of Surgery, Austin Health, The University of Melbourne, Heidelberg, VIC, Australia
}

(c) 2011 Ager et al; licensee BioMed Central Ltd. This is an Open Access article distributed under the terms of the Creative Commons Attribution License (http://creativecommons.org/licenses/by/2.0), which permits unrestricted use, distribution, and reproduction in any medium, provided the original work is properly cited. proliferative, proinflammatory, and angiogenic effects of ANG II $[4,5]$.

The RAS also has an 'alternative' pathway which counteracts many of the actions induced by ANG IIAT1R signalling. The alternative ANG II receptor (the AT2R) generally exerts actions antagonistic to the AT1R including inhibition of proliferation and promotion of apoptosis [6]. ACE2, a homologue of ACE, generates a second RAS peptide, ANG-(1-7), directly from ANG II. ANG-(1-7), through its specific receptor MasR, also appears to counteract many of the actions induced by the classical AT1R/ANGII RAS pathway [7]. Activation of the alternative ANG II receptor, the AT2R, has been shown to inhibit tumour growth (although to lesser 
extent then either irbesartan or captopril)[5]. ANG-(1-7) can also be infused to reduce tumour growth in several experimental cancer models $[8,9]$. Two independent Phase I clinical trials are examining ANG-(1-7) [10] and AT1R blockade [11] in the treatment of various solid tumours.

Given the counter-regulatory actions of the classical and alternative RAS pathways we hypothesized that combining inhibition of the classical RAS (AT1R blockade or ACE inhibition) with activation of the alternative RAS (ANG-(1-7) infusion or AT2R activation) would synergistically inhibit tumour growth.

\section{Methods}

\section{In vivo model and cell lines}

The mouse colorectal cancer (MoCR) cell line used for in vivo experiments was harvested from a dimethylhydrazine-induced colon carcinoma in a CBA mouse at a stage known to metastasise to the liver [12]. Liver metastases were induced as described previously $[3,12]$. Briefly, $25000 \mathrm{MoCR}$ cells were injected into the spleen of 6 to 8 week old male CBA mice and, after 3 minutes, the spleen removed to confine metastases to the liver. A minimum of 5 animals per group were used, in treatments inducing fewer tumours sample size was increased to 10. All experiments were approved by the Austin Health Animal Ethics Committee. Liver samples were collected and fixed in fresh 4\% PFA.

\section{Drugs/agents and treatments}

In vivo treatments included ANG-(1-7) (Auspep, 2588; $24 \mu \mathrm{g} / \mathrm{kg} / \mathrm{hr}$ ), CGP42112A (AT2R agonist, SigmaAldrich, C160; $0.6 \mu \mathrm{g} / \mathrm{kg} / \mathrm{hr}$ ), and/or telmisartan (AT1R blocker, Sigma-Aldrich, T8949; $12.5 \mu \mathrm{g} / \mathrm{kg} / \mathrm{hr}$ ) infusion (Alzet $^{\circledR}$ osmotic mini pumps 1004) or s.c. daily injections of irbesartan (AT1R antagonist, Bristol-Myers Squibb) at $50 \mathrm{mg} / \mathrm{kg}$. Captopril was given as daily i.p. injections of $750 \mathrm{mg} / \mathrm{kg}$ (Sigma-Aldrich, 21751). Doses were based on previously published studies [3,5,13-15]. The solubilising agent (saline or methyl cellulose) provided a control. Treatments continued from the time of tumour induction to tissue collection at day 21 .

\section{Immunohistochemistry}

AT1R (rabbit polyclonal against human, Santa Cruz, sc1173), proliferation (Ki67; rat monoclonal anti-mouse, Thermoscientific, RM-9106-S1), apoptosis (active caspase 3; rabbit polyclonal anti-human, R\&DSystems AF835), angiogenesis (CD34, neovascularisation marker; monoclonal rat anti-mouse, Abd Serotec MCA18256), and VEGF (rabbit polyclonal anti-human, CalBiochem, PC315) were assessed in PFA-fixed paraffin embedded tissues. Specificity of AT1R and VEGF antibodies was confirmed by western blot (data not shown). AT1R was used at a concentration of $0.5 \mu \mathrm{g} / \mathrm{ml}$, Ki67 at 1:100 dilution (dilution provided with manufacturer's datasheet), active caspase- 3 at $1.0 \mu \mathrm{g} / \mathrm{ml}, \mathrm{CD} 34$ at $0.1 \mu \mathrm{g} / \mathrm{ml}$, and VEGF at $1.5 \mu \mathrm{g} / \mathrm{ml}$. Non-immunized rabbit IgG (Santa Cruz, sc-2027) at an equivalent concentration to the primary target antibody was used as a negative control. Endogenous peroxidases were blocked with $3 \% \mathrm{H}_{2} \mathrm{O}_{2}$ and non-specific binding inhibited with $10 \%$ normal goat serum (Zymed, 01-6201). Slides were incubated with primary antibodies at $37^{\circ} \mathrm{C}$ for 1 hour and then $4^{\circ} \mathrm{C}$ overnight. Slides were then incubated with the secondary antibody (Dako Envision ${ }^{+}$Goat anti-rabbit HRP secondary 4011 for AT1R, Ki67, caspase 3, and VEGF and the Rat on Mouse AP-polymer kit (Biocare Medical; $\mathrm{RT} 518 \mathrm{H}$ ) for CD34 for 1 hour at $37^{\circ} \mathrm{C}$ before visualisation with DAB or, for CD34, Vulcan fast red (Applied Medical FR805H). Slides were counterstained with Mayer's haematoxylin.

Images of stained tumours were taken using digital light microscope (Nikon Coolscope ${ }^{\circledR}$, Nikon Corporation, Japan) at between 40x and 400x magnification (with a scale bar for size calibration) and were analysed using Image-Pro plus (version 5). Depending on the animal and its tumour load, between 10 and 30 images across 1 to 5 tumours were taken for analysis. AT1R staining was assessed in control tissues, while VEGF, Ki67, caspase3, and CD34 were all examined in control and irbesartan treated tissues. Where possible, the number of positive cells out of the total number of cells was used to measure changes in protein levels/content. A scoring system was used to quantify differences in the strength and abundance of AT1R and VEGF staining in tumours. The intensity of immune-reactive staining was evaluated subjectively (by two independent researchers) using the intensity of immunoreactive colour development as an measure of relative protein content. Strong staining of many/most cells was given a grade of 5 reducing to no staining (0). Only areas of viable tumour were considered in analysis. A subpopulation of intense VEGF expressing infiltrating cells was also counted and is expressed as the number of positive cells per area tumour. Ki67 and active caspase3 were assessed as both the area of proliferating tumour (4x magnification) and the number of proliferating cells per area tumour or liver (20x magnification). CD34 was assessed as the length of positively stained endothelium per tumour area and provide an indication of the angiogenic potential of these tumours.

\section{Statistical analyses}

Quantitative data are presented as mean \pm SEM or boxplots showing the minimum value, first quartile, median, third quartile and maximum value. Statistical analyses were conducted using SPSS (Statistical Package for the 
Social Sciences, version 17, USA) or Microsoft Excel (2003). Normally distributed data were assessed by ANOVA. Mann-Whitney U test were used for nonparametric data sets. A probability $(P)$ value of less than 0.05 was considered as statistically significant.

\section{Results \\ Captopril and ANG-(1-7) inhibited tumour burden while AT1R blockade had no significant effect}

Mice were induced with CRC liver metastases and treated with irbesartan, ANG-(1-7), or captopril while control received vehicle (PBS or methyl cellulose). Liver-tobody weight ratio was used as a measure of liver tumour burden. No significant difference between the control and the irbesartan treated group was detected (Figure 1A). Given that we had previously found irbesartan inhibited tumour growth in this model [3] we decided to test another AT1R blocker, telmisartan (also presented in Figure 1A); however, again there was no significant difference between control and telmisartan treated groups. We also decided to examine tumour burden by quantitative stereology as this provides a more sensitive measure of tumour load. However, as with the liver to body weight ratio, no significant difference was seen in the response of tumours to either irbesartan (Figure 1B) or telmisartan (data not shown), while we confirmed the ANG-(1-7)-associated reduction in tumour growth $(P=0.00263$, $\mathrm{t}$-test unequal variance) (Figure 1B).

ACE inhibition with captopril was found to retain its anti-tumour activity (Figure 1A). Captopril-treated animals had significantly lower liver-to-body weight ratios $(0.312 \pm 0.010)$ compared to the control group with $(0.139 \pm 0.011)\left(P=2.907 \times 10^{-8}\right.$, $\mathrm{t}$-test equal variance $)$. The level of tumour inhibition achieved by captopril treatment in the current experiment is comparable to our previously published studies $[2,3]$.

\section{Combination therapies targeting the RAS did not improve upon levels of tumour inhibition achieved through single arm therapies}

Although irbesartan alone did not significantly inhibit tumour growth, we continued with the initial aim of combining AT1R blockade and/or captopril with ANG(1-7) infusion. As with the single captopril treatment, combined treatment with captopril and ANG-(1-7) significantly inhibited tumour growth (Figure 2). However, there was no difference between the level of inhibition achieved by captopril alone (liver to body weight ratio of $0.139 \pm 0.11)$ compared to combined captopril plus ANG-(1-7) (liver to body weight ratio of $0.149 \pm 0.02$ ). Irbesartan plus ANG-(1-7) failed to decrease tumour burden (Figure 2); this is despite the fact that ANG-(17) alone resulted in a reduction in liver to body weight ratio (Figure $1 \mathrm{~A}$ and $1 \mathrm{~B}$ ). Similarly, AT2R activation with CGP42112A combined with AT1R blockade by telmisartan failed to decrease tumour burden despite previously reported CGP42112A-induced inhibition of CRC liver metastases [5].

\section{AT1R immunostaining increased in cancer cells from irbesartan-insensitive compared to irbesartan-sensitive animals}

While irbesartan was previously found to inhibit tumour growth in this mouse model of CRC liver metastases, in the current experiment, it failed to reduce liver tumour burden despite the same experimental protocols being employed. We hypothesized that this difference was likely due to either to the animals used, which although of the same strain, sex, and age were obtained 2 year apart, or to the cell line, which again may have acquired changes over the intervening years between experiments.

Immunohistochemistry was used to assess the relative level and extent of AT1R expression in CRC-derived tumours growing in the liver from both studies. While no obvious difference was seen in the liver surrounding tumours, tumours from the current study showed a marked upregulation of AT1R expression. AT1R staining in cancer cells was found to be higher in the irbesartan-insensitive animals compared to the irbesartansensitive animals (Additional file 1 and Figure 3). Pairwise comparisons between tumours of different sizes all showed higher AT1R expression in irbesartan-insensitive tumours $\left(\mathrm{AT} 1 \mathrm{R}^{\mathrm{HI}}\right.$ ) compared to irbesartan-sensitive tumours $\left(\mathrm{AT} 1 \mathrm{R}^{\mathrm{LOW}}\right.$ ) (small tumours: 3 vs $2, P=0.002$; medium tumours score: 4 vs $2, P<0.0001$; large tumours: 4 vs $2, P<0.0001$ respectively, Mann-Whitney U test).

Within the liver there were AT1R positive cells in both irbesartan-sensitive and irbesartan-insensitive animals. A majority of these cells co-stain with the F4/80 macrophage marker (data not shown). The number and intensity of non-paranchymal, non-cancer cell associated AT1R staining did not appear to differ between the irbesartan-sensitive and the irbesartan-insensitive animals.

\section{Proliferation of cancer cells was markedly increased in the $A T 1 R^{\mathrm{HI}}$ cancers}

$\mathrm{AT} 1 \mathrm{R}^{\mathrm{HI}}$ tumours were associated with a marked increase (between a $17 \%$ to $32 \%$ increase) in proliferation compared to AT1 $\mathrm{R}^{\mathrm{LOW}}$ tumours (Figure $4 \mathrm{~A}$ and $4 B)$. This was significant for all comparisons $(P \leq$ 0.0052 , t-test) when measured as the number of proliferating cells, but was a trend when measured as the area of proliferation $\left(\mathrm{AT} 1 \mathrm{R}^{\mathrm{HI}}\right.$ control compared to AT1 $\mathrm{R}^{\mathrm{LOW}}$ control, Figure 4A). Neither hi- nor low-AT1R-expressing tumours showed a reduction in proliferation with irbesartan treatment. This corresponds with previously 
A) Tumour burden assessed by liver to body weight ratio

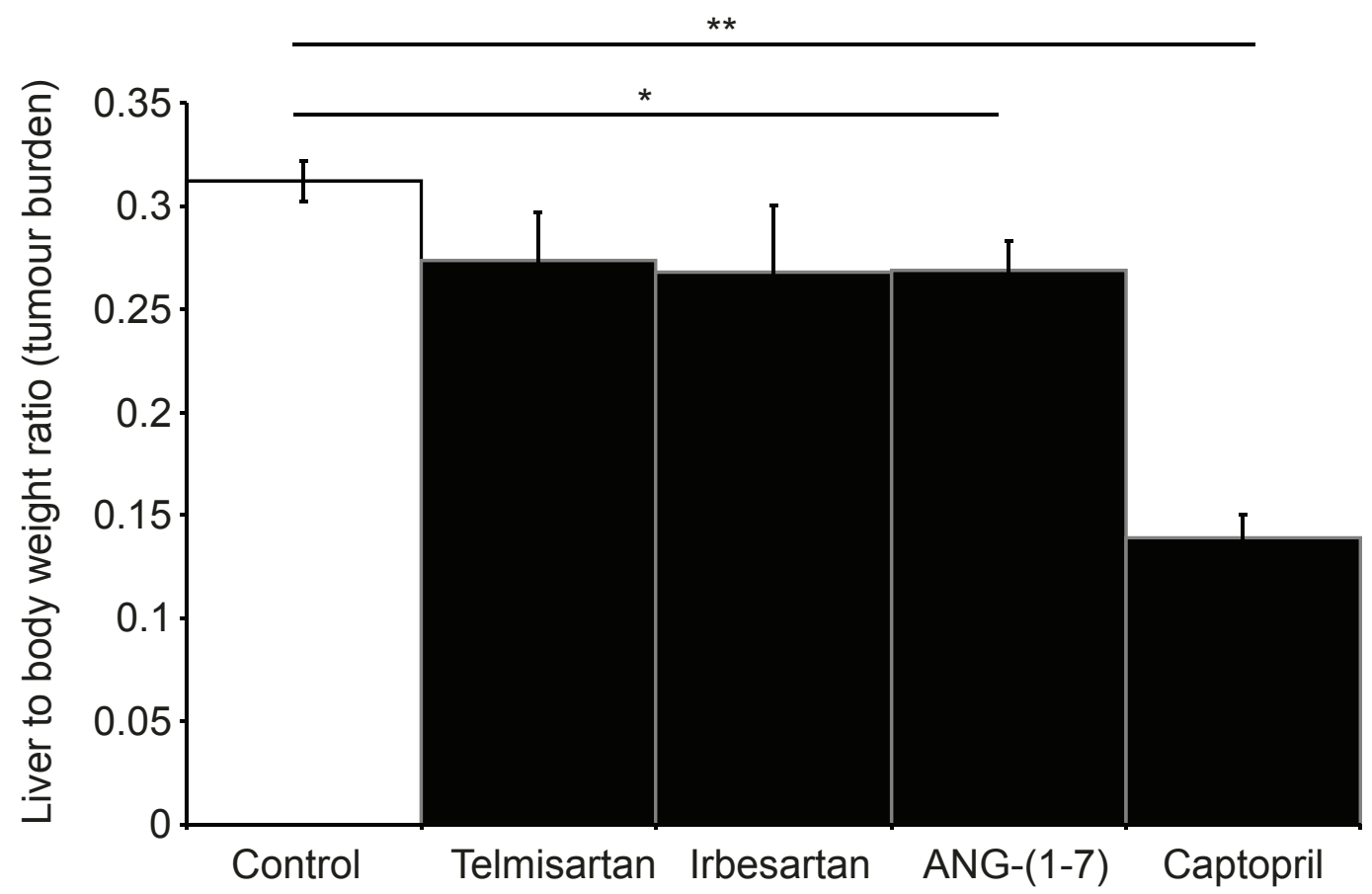

B) Tumour burden assessed by stereology

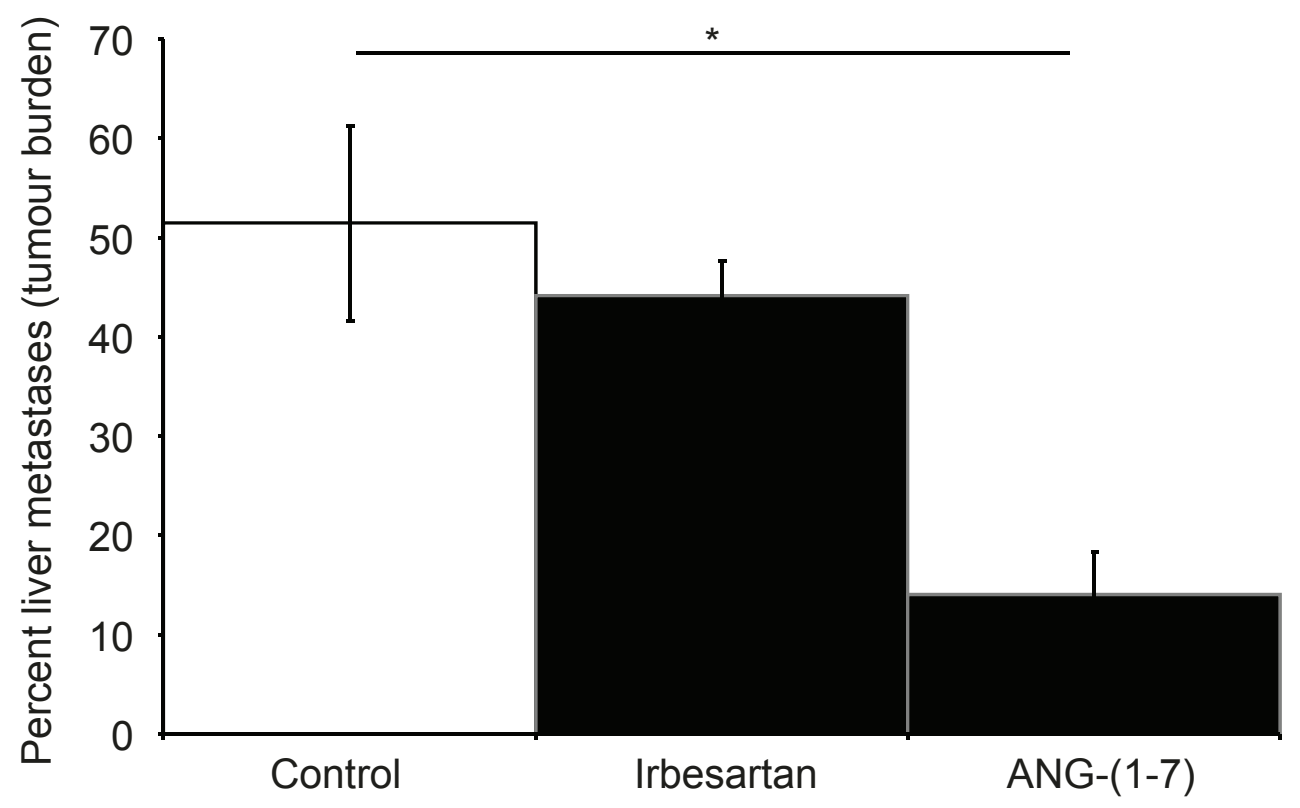

Figure 1 Liver to body weight ratio 21 days after tumour inoculation with continuous RAS-targeted single treatments (A). Percent liver metastases for irbesartan and ANG-(1-7) or control (untreated) animals from the same study as above (B). Telmisartan and irbesartan are AT1R blockers, while captopril is an ANCE inhibitor. $n=5$ or 6 for each group. Significant $P$ values between 0.01 and 0.05 are shown with an *, those less than 0.01 are shown with ${ }^{*}$. Data are presented as mean \pm S.E.M. 


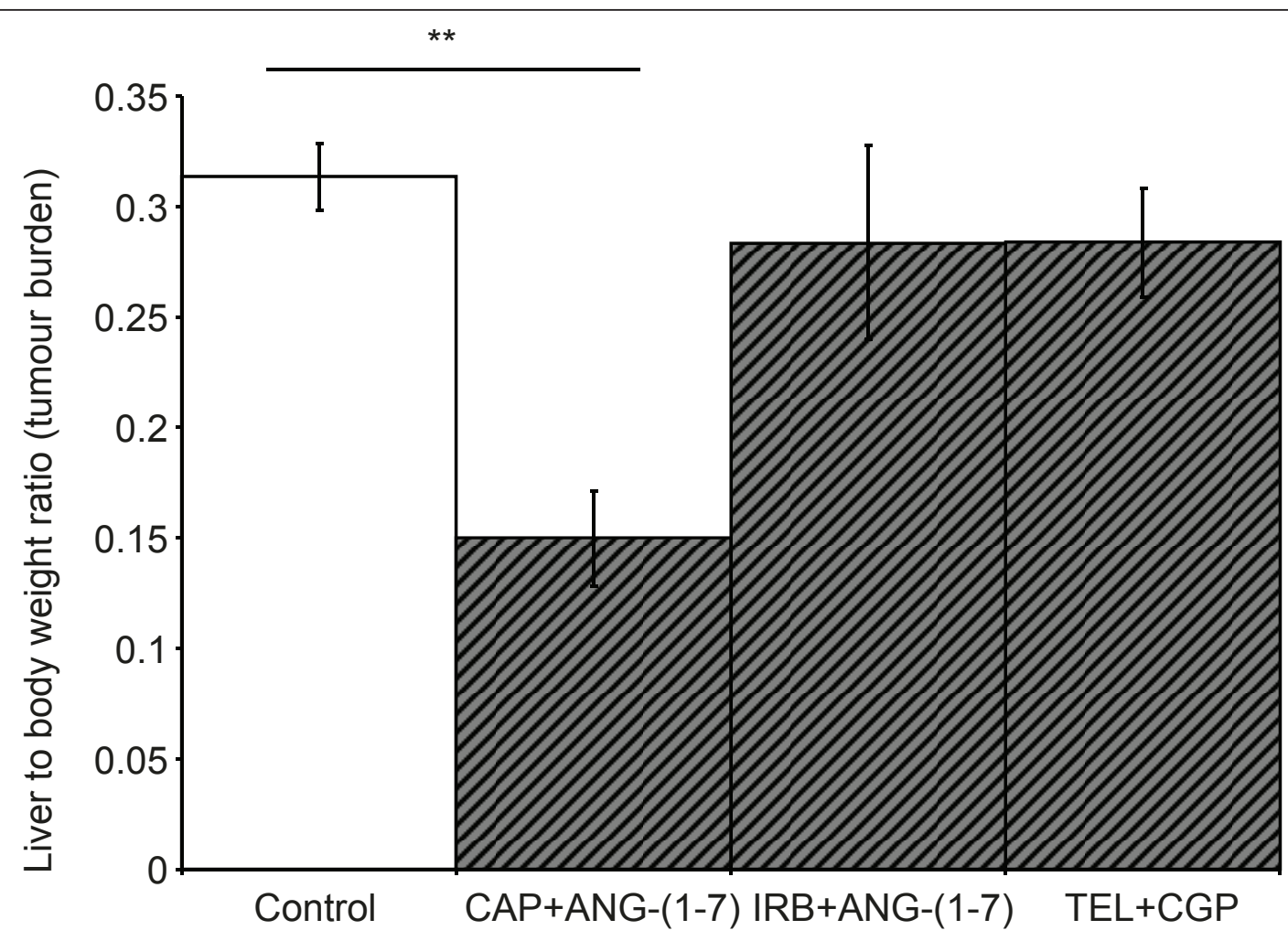

Figure 2 Liver to body weight ratio 21 days after tumour inoculation with continuous RAS-targeted combined treatments. CAP, captopril; IRB, irbesartan; TEL, Telmisartan. $n=5$ or 6 for each group. Significant P values between 0.01 and 0.05 are shown with an *, those less than 0.01 are shown with ${ }^{* *}$. Data are presented as mean \pm S.E.M.

unpublished data from our group which showed an increase in apoptosis with irbesartan, but no change in proliferation (Additional file 2).

In contrast to the increase in cancer-cell proliferation by $\mathrm{AT}_{1} \mathrm{R}^{\mathrm{HI}}$ tumours, the liver surrounding these tumours had fewer $(P \leq 0.0327$, t-test) proliferating cells compared to animals bearing AT1 ${ }^{\mathrm{LOW}}$ tumours (Figure $4 C)$. Moreover, there was a marked reduction $(P \leq$ 0.0002 , t-test) in the number of these proliferating cells with irbesartan treatment in the AT1R ${ }^{\mathrm{LOW}}$, but not the $A T 1 R^{\mathrm{HI}}$ tumour-bearing animals. It is not clear what type of cell is represented, but both non-paranchymal and paranchymal cells were present in the proliferating population and based on morphology and location relative to the sinusoids, these cells are likely to be hepatic stellate cells or metastasizing tumour cells. Indeed, in our previous study we showed that irbesartan could reduce the number of metastatic lesions [3].

Apoptosis was increased by irbesartan treatment in both $\mathrm{AT}^{\mathrm{R}} \mathrm{R}^{\mathrm{HI}}$ and $\mathrm{AT} 1 \mathrm{R}^{\mathrm{LOW}}$ tumours

Irbesartan treatment significantly increased the number of apoptotic cells in both $\mathrm{AT}_{1} \mathrm{R}^{\mathrm{HI}}$ tumours $(P=0.0291$, t-test) and AT1 ${ }^{\text {LOW }}$ tumours $(P=0.0144)$ (Figure 5$)$. Although this increase was not as great in the AT1 ${ }^{\mathrm{HI}}$ tumours compared to that seen in the $A T 1 R^{\mathrm{LOW}}$ tumours. Both treated and non-treated AT1 $\mathrm{R}^{\mathrm{HI}}$ tumours showed higher levels of apoptosis than their corresponding $\mathrm{AT}_{1} \mathrm{R}^{\mathrm{LOW}}$ tumours $(P \leq 0.000$, t-test $)$.

\section{VEGF expression was higher in $A T 1 R^{H I}$ compared to} AT1R ${ }^{\text {LOW }}$ tumours

Similar to cancer-cell proliferation, cancer cell VEGF expression was significantly higher in $A T 1 R^{\mathrm{HI}}$ tumours compared to their corresponding $\mathrm{AT}^{\mathrm{T}} \mathrm{R}^{\mathrm{LOW}}$ tumours $(P$ $\leq 0.000$, t-test) (Figure 6A). Interestingly, the median level of VEGF intensity was higher for both irbesartan treated groups (although this failed to reach significance).

While cancer cell-associated VEGF was higher in the AT1 $\mathrm{R}^{\mathrm{HI}}$ tumours $(P \leq 0.0123$, t-test $)$, the number of intense-staining infiltrating cells was highest in the $\mathrm{AT} 1 \mathrm{R}^{\mathrm{LOW}}$ tumours and this was decreased by irbesartan treatment $\left(P=1.109 \times 10^{-5}\right)$ (Figure 6B).

CD34+ (angiogenic) vessels were increased in $\mathrm{AT}^{\mathrm{HI}}$ tumours and decreased by irbesartan treatment in both $\mathrm{AT}^{\mathrm{H}} \mathrm{R}^{\mathrm{HI}}$ and $\mathrm{AT}^{\mathrm{R}} \mathrm{R}^{\mathrm{LOW}}$ tumours

Our unpublished data from previous studies (Additional file 3) showed that ANGII infusion increased the length 


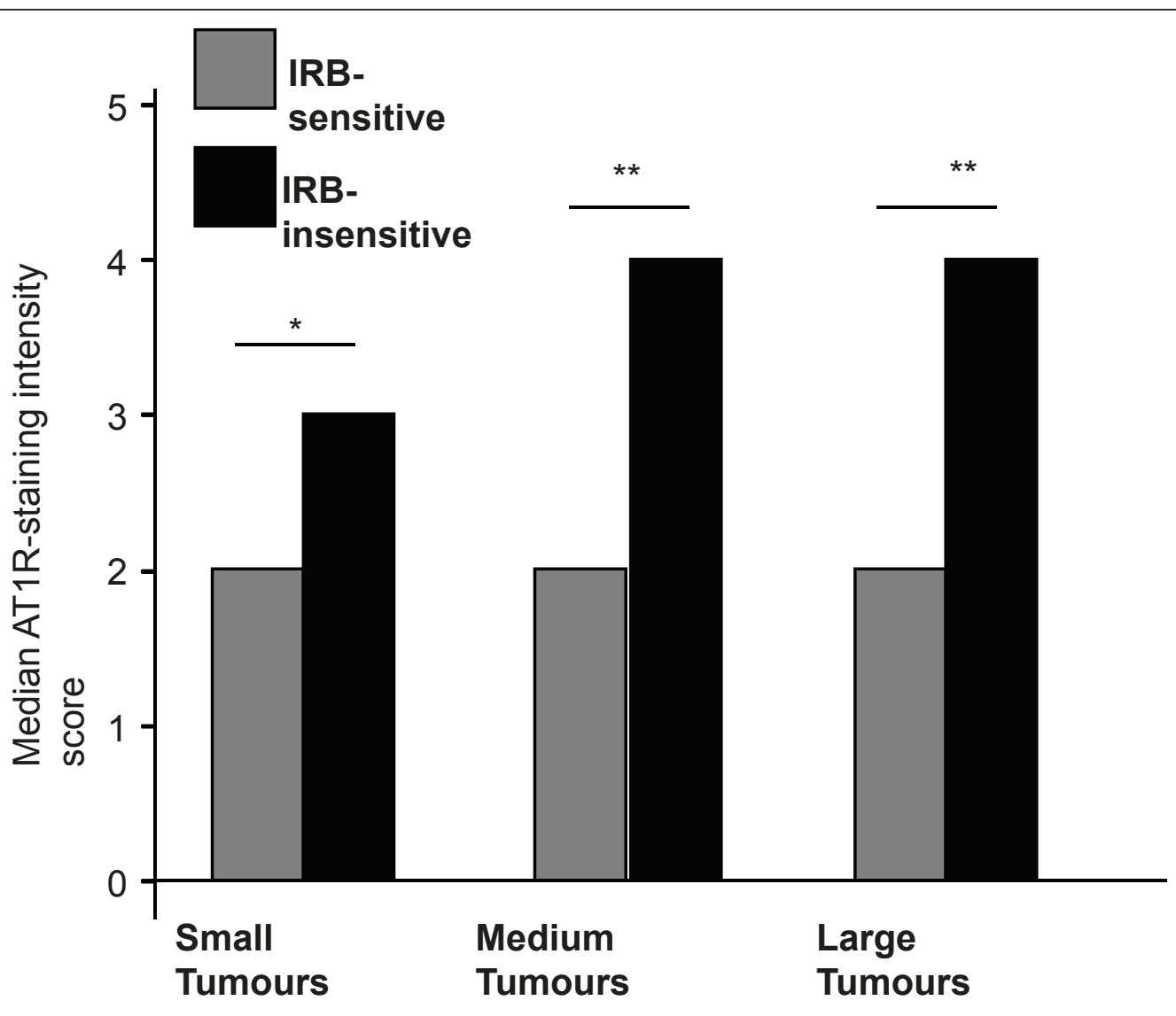

Irbesartan-sensitive

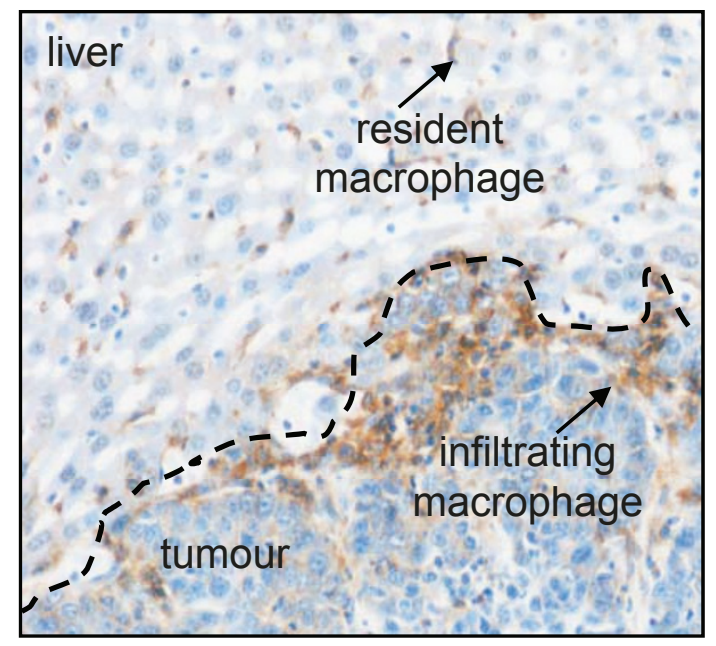

Irbesartan-insensitive

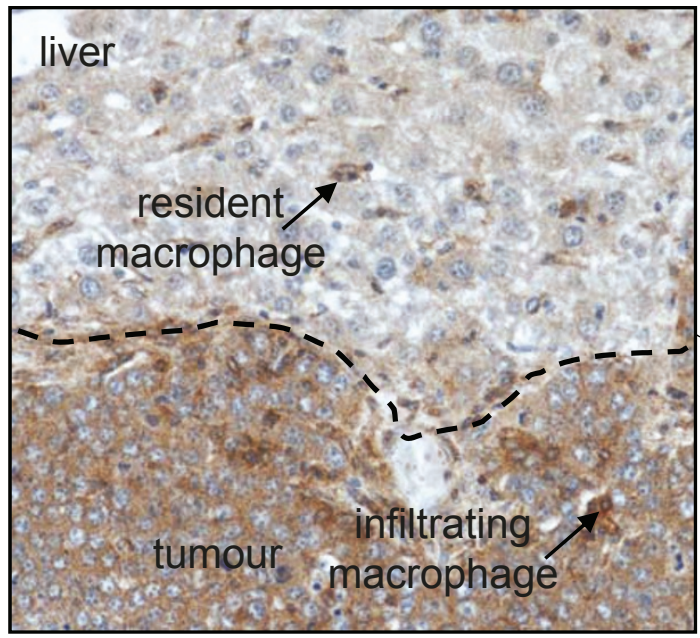

Figure 3 Intensity of AT1R staining 21 days after tumour inoculation in untreated (control) animals with tumours showing sensitivity to irbesartan or insensitive to irbesartan treatment. Tumours were grouped into small $(\leq 250 \mu \mathrm{m})$, medium $(250 \mu \mathrm{m}$ to $1000 \mu \mathrm{m})$, or large $(\geq 1000 \mu \mathrm{m})$ in diameter. Representative images are shown below. $\mathrm{n}=5$ or 6 for each group except for AT1R ${ }^{\text {Low }}$ tumours which, because of the reduced tumour load, had additional samples ( $n$ of 10). The entire tumour was imaged for analysis. Significant $P$ values between 0.01 and 0.05 are shown with an *, those less than 0.01 are shown with **. Data are presented as mean \pm S.E.M. 


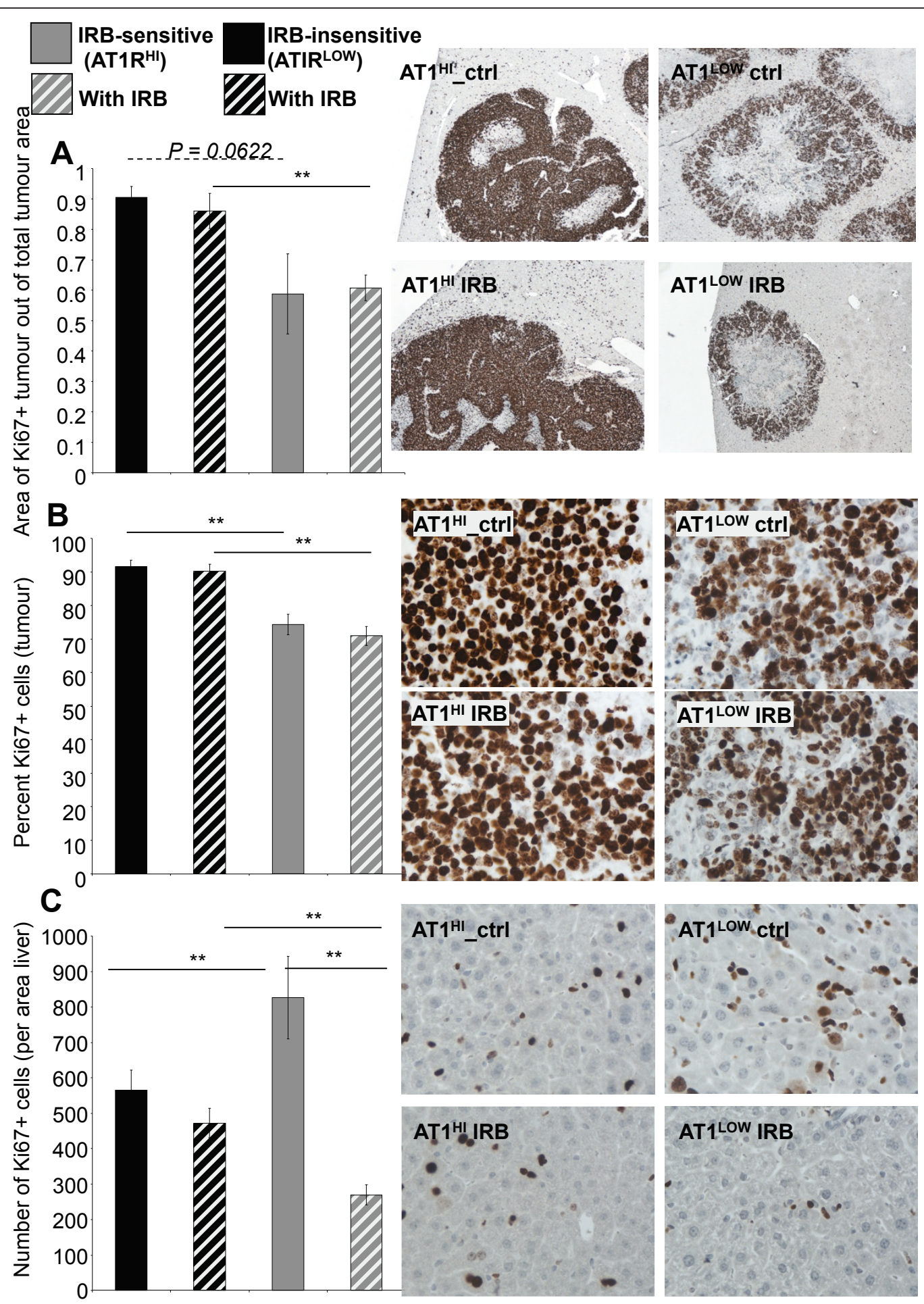

Figure 4 Area of proliferating tumour 21 days after tumour inoculation in irbesartan sensitive (IRB-sensitive; AT1R ${ }^{\text {LOW }}$ ) and insensitive (IRB-insensitive; $\mathbf{A T 1} \mathbf{R}^{\mathrm{HI}}$ ) tumours either treated with irbesartan (IRB) or untreated. (A). The percent of Ki67 positive (proliferating) cells per tumour area (measured at 400x magnification) (B). The number of Ki67 positive cells in the liver surrounding tumour (measured at 400x magnification) (C). $n=5$ or 6 for each group except for AT1R ${ }^{L O W}$ tumours which, because of the reduced tumour load, had additional samples (n of 10). Between 10 and 30 images were taken from each mouse across 1 to 5 tumours (dependent on tumour load). Representative images for each graph are shown to the right. Significant $P$ values between 0.01 and 0.05 are shown with an ${ }^{*}$ and solid line, those less than 0.01 are shown with **, while those of interest with $\mathrm{P}$ values between 0.1 and 0.05 are indicated with a dotted line and no *, but with $\mathrm{P}$ value shown. Data are presented as mean \pm S.E.M. 


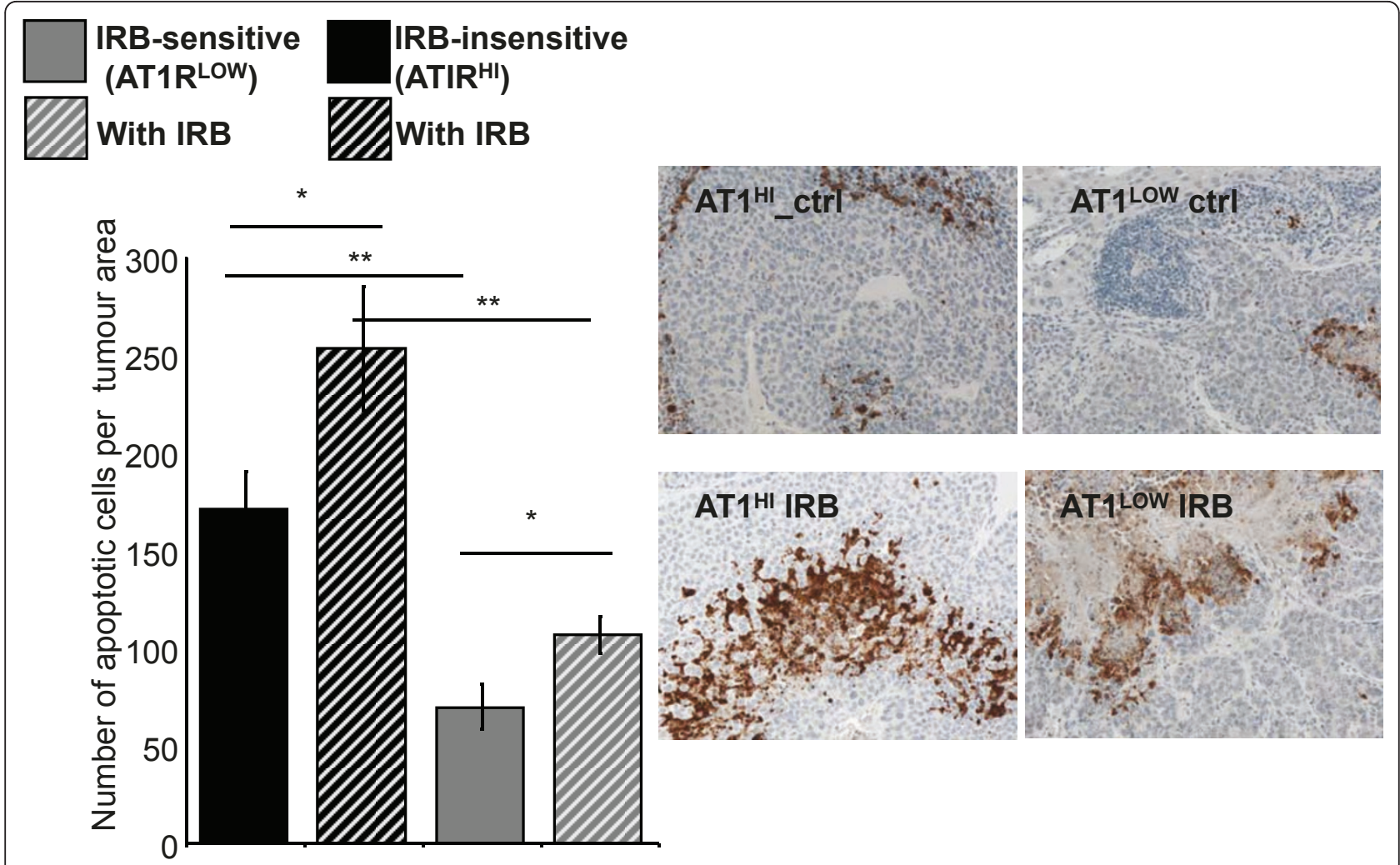

Figure 5 The number of apoptotic cells per tumour area (measured at 200x magnification). $n=5$ or 6 for each group except for $A T 1 R^{L O W}$ tumours which, because of the reduced tumour load, had additional samples (n of 10). Between 10 and 30 images were taken from each mouse across 1 to 5 tumours (dependent on tumour load). Representative images are shown to the right. Significant P values between 0.01 and 0.05 are shown with an *, those less than 0.01 are shown with **. Data are presented as mean \pm S.E.M.

of CD34+ endothelium in tumours while irbesartan decreased CD34+ endothelium compared to tumours from control (untreated) animals. Here we also found that irbesartan decreased the length of CD34+ endothelium in tumours and that this was the case regardless of cancer cell AT1R expression level (Figure 7). Similar to the increase in apoptosis, the decrease in CD34 staining was greatest in the AT $1 R^{\mathrm{LOW}}$ tumours $(P=0.0267$ for AT1 ${ }^{\mathrm{HI}}$ compared to $P=0.0179$ for $\mathrm{AT} 1 \mathrm{R}^{\mathrm{LOW}}$ tumours). However, both treated and non-treated $\mathrm{AT} 1 \mathrm{R}^{\mathrm{HI}}$ tumours had more angiogenic vessels than AT1R ${ }^{\text {LOW }}$ tumours $(P \leq 0.000$, t-test $)$.

\section{Discussion}

Blockade of the classical RAS through AT1R blockade or ACE inhibition reduces tumour growth in several experimental mouse models of cancer $[2,3,16,17]$. Conversely activation of the alternative RAS, through ANG(1-7) infusion [8] or AT2R activation [5], can also reduce tumour growth. We sought to determine if greater inhibition of tumour growth could be achieved through dual targeting of the RAS - inhibition of the classical RAS with simultaneous activation of the alternative RAS. While, we were unable to show any benefit for combined RAS treatments, we found that an upregulation of the AT1R by the cancer cell line rendered them insensitive to AT1R blockade. These same cells, however, remained sensitive to ACE inhibition, suggesting significant differences in the molecular mechanisms by which these agents inhibit tumour growth.

The anti-tumour effects of AT1R blockers have been well documented $[3,18,19]$. However, in our experiment, irbesartan, when used alone or in combination with ANG-(1-7) infusion, failed to decrease tumour growth. We also tested the potent AT1R blocker telmisartan, which has been utilised as an anti-cancer agent in other experimental models where it was shown to be effective $[16,20,21]$, but again we found no benefit of treatment compared to control animals. The lack of an effect of AT1R blockade also contradicts a previous study conducted in our laboratory using the same experimental protocols [3].

To further investigate this finding we performed immunohistochemical analysis on tumours from both studies (for which tissues had been collected in the 


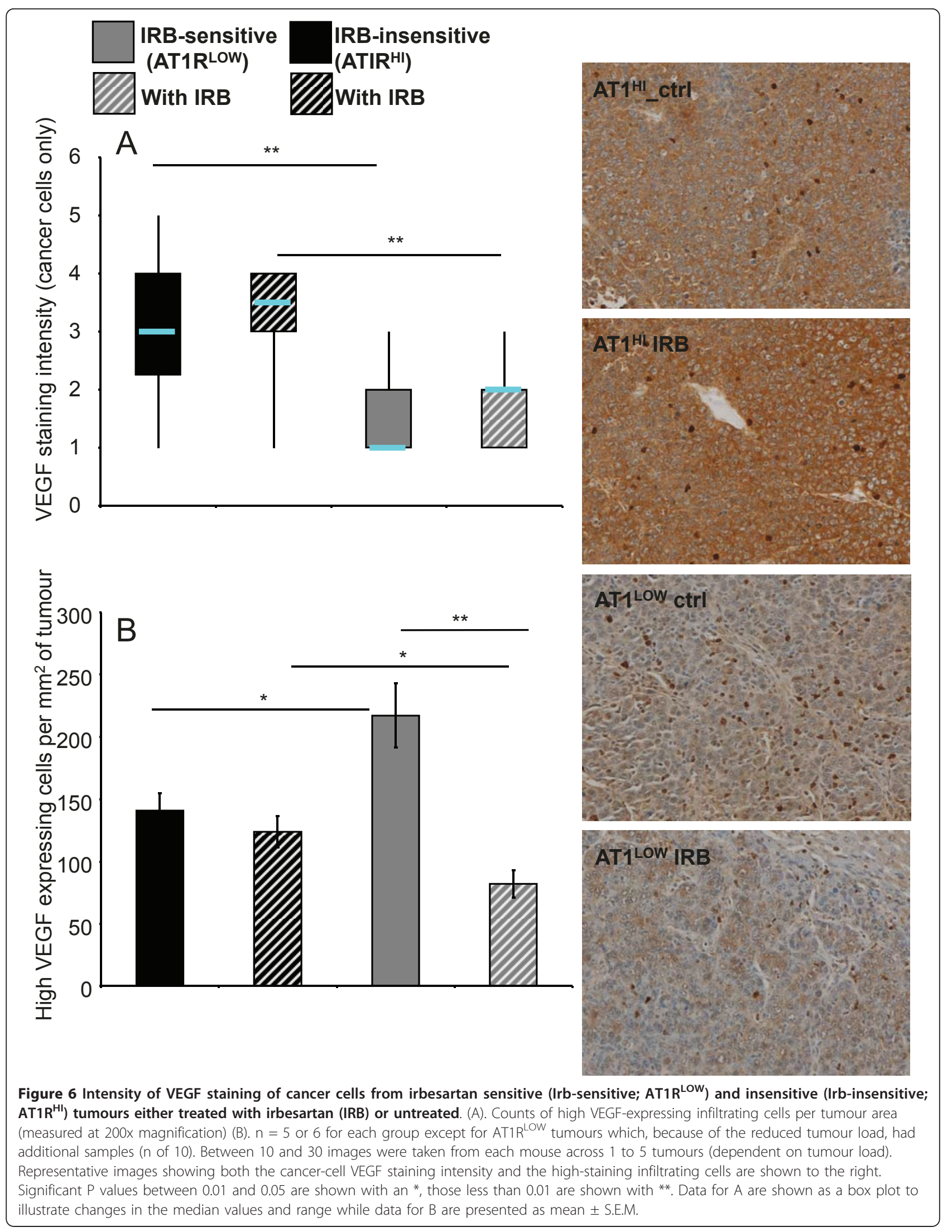




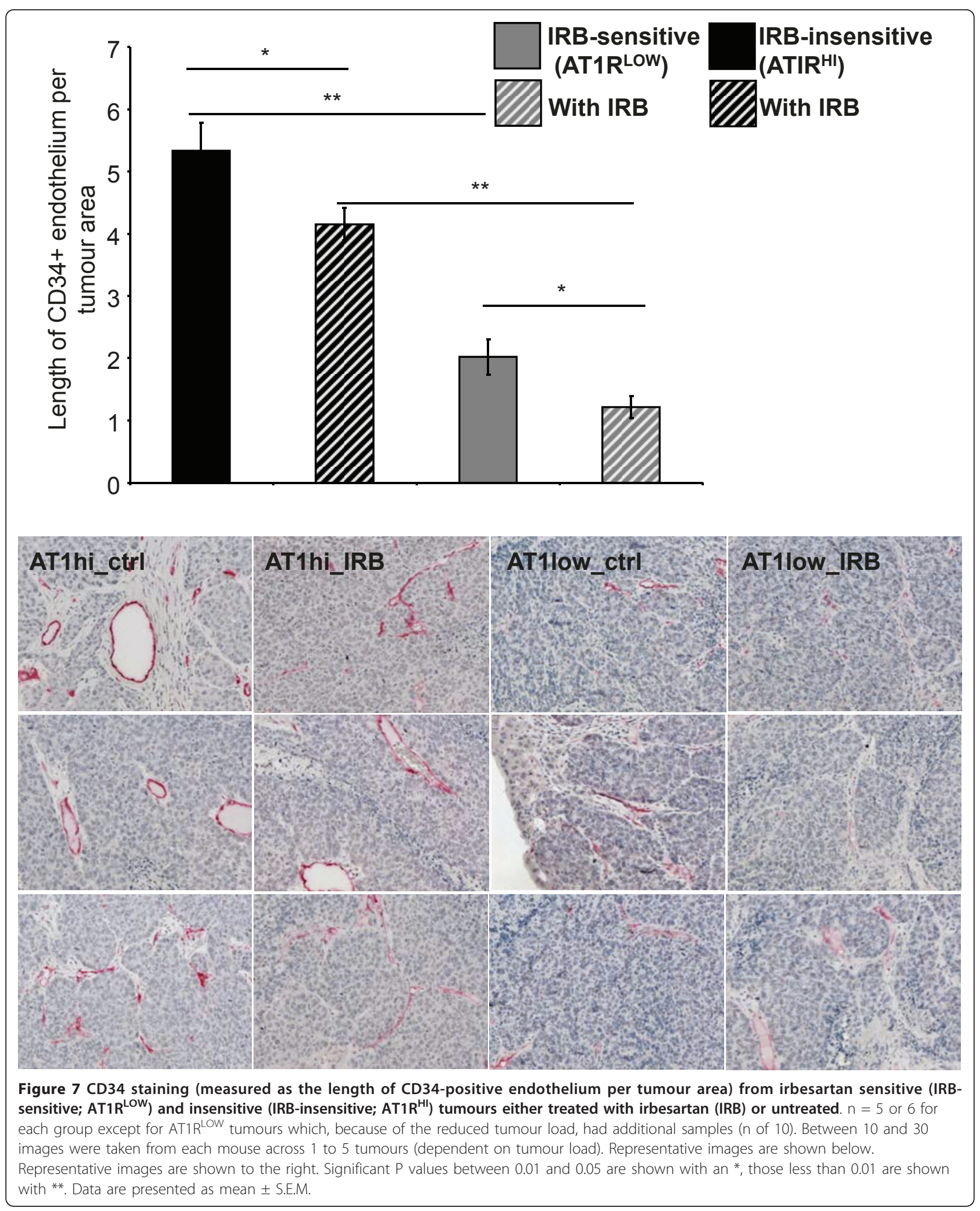


same experimental manner). AT1R expression by cancer cells from animals insensitive to AT1R blockade showed a marked increase in immunohistochemical staining compared to tissues collected from our previous irbesar$\tan$ sensitive animals. These results suggest that cancer cells could be rendered insensitive to AT1R blockade through an upregulation of the AT1R. Moreover, despite strong AT1R expression by liver macrophages described here and elsewhere [22] and the importance of macrophages during tumour progression [23], our results suggest that cancer cell AT1R expression can overcome any macrophage-mediated antitumour activity associated with AT1R blockade.

The AT1R is also known to regulate angiogenesis, migration, and apoptosis all of which could contribute to regulating tumour growth $[24,25]$. Thus, it is likely that the upregulation of AT1R is, at least in part, responsible for the resistance of these cells to AT1R blockade. In support of this we found that high AT1R-expressing tumours had increased cancer cell proliferation, higher levels of cancer cell-associated VEGF and tumour-associated angiogenesis. Interestingly, irbesartan lead to a slight increase in VEGF levels in both tumour types. This is in contrast to some studies of breast cancer [26,27], but supports our previous study which showed that, in these tumours, activation of the AT2R increased VEGF expression [5]. Clere et al. (2010) similarly found that in fibrosarcoma, the AT2R could promote cancer cell VEGF production [28]. AT2R expression has also been documented in blood vessels of human pituitary adenomas [29] and both the AT1R and AT2R stimulate VEGF secretion by rat pituitary tumour cells [30]. Thus, it would appear that in at least some cancer-associated circumstances the AT2R can also be proangiogeneic. However, angiogenesis (as measured by CD34 staining) was decreased by irbesartan and this appeared to be associated with a decrease in the number of infiltrating VEGF-expressing cells.

We also report an increase in apoptosis and a decrease in angiogenic vessel formation associated with irbesartan, and while still present in AT1 ${ }^{\mathrm{HI}}$ tumours, these effects were less than that seen for AT1 $\mathrm{R}^{\mathrm{LOW}}$ tumours. Additionally, while it is apparent that cancer-cell AT1R expression has a direct effect on cancer cell proliferation, VEGF-expression, and apoptosis, we also saw marked changes in the surrounding liver and in cells infiltrating the tumours. In particular, irbesartan sensitive tumours $\left(\mathrm{AT} 1 \mathrm{R}^{\mathrm{LOW}}\right.$ ) when untreated (i.e. control animals) were associated with higher numbers of VEGF-infiltrating cells in tumours and higher numbers of proliferating cells in the liver surrounding tumours. Both of these phenomena were reduced by irbesartan treatment. These results would suggest that while tumour resistance was conferred by higher cancer cell-AT1R expression (and that this was associated with certain growth advantages such as increased proliferation and reduced treatment-associated apoptosis), the non-parenchymal cells in the liver may be important in determining the response of low AT1R-expressing tumours to irbesartan.

In contrast to AT1R blockade, ACE inhibition by captopril maintained its effectiveness, consistent to the previous findings [3]. This suggests that ACE inhibitors, other than blocking the production of ANG II which subsequently binds AT1R, also generate anti-tumour effects through non-ANG II mediated pathways. Indeed this has been documented in several studies where matrix metalloproteinases and vascular endothelial growth factor expression have been recognised as nonRAS dependent targets of ACE inhibition [31-33].

\section{Conclusions}

This research initially set out to determine if AT1R blockade or ACE inhibition in combination with either ANG-(1-7) or AT2R activation could lead to synergistic inhibition of CRCLM. While we failed to show any benefit of combined targeting of the RAS, our results were fortuitous in providing insight into both the importance of differences between the mechanisms of action of ACE inhibition and AT1R blockade as well as the potential of RAS expression as a biomarker.

\section{Additional material}

Additional file 1: Table indicating the intensity of AT1R staining by different CRC liver metastases. AT1R staining scores in tumours from animals showing insensitivity to irbesartan treatment and animals in which irbesartan successfully inhibited tumour growth (irbesartansensitive). All treatment protocols including agent dose and timing, cancer cell numbers (and type -expecting AT1R expression as described below) and method of induction, as well as tissue collection and processing were identical between experiments.

Additional file 2: Figure showing proliferation and apoptosis of cancer cells from irbesartan-sensitive tumours. Proliferation of cancer cells from irbesartan-sensitive tumours as measured by PCNA immunostaining (A). Apoptosis, measured by the percent of active-caspase3positively stained tumour area, of cancer cells in the same tumour/liver samples as in $A(B)$.

Additional file 3: Angiogenesis (CD34 staining) of irbesartansensitive tumours. Degree of angiogenic vessel formation (as measured by CD34immuno- staining from animals bearing Irbesartan-sensitive tumours

\section{Abbreviations}

ANG II: angiotensin II; ACE: angiotensin converting enzyme; AT1R angiotensin II type 1 receptor; AT2R: angiotensin II type 2 receptor; CRC: colorectal cancer; VEGF: vascular endothelial growth factor; RAS: renin angiotensin system.

\section{Acknowledgements}

This work was supported by Cancer Australia's Priority-driven Collaborative Cancer Research Scheme and was co-funded by Cancer Australia and Cure Cancer Australia Foundation. We would also like to thank the CanToo fund raisers. Initial funding was provided by the Cancer Council of Victoria. Dr Ager is supported by an NHMRC Post-doctoral Training Award. Ms Shu-wen 
Wen and Dr Jaclyn Neo are supported by an Australian Rotary Health Research Fund PhD Scholarship.

\section{Authors' contributions}

EA conceived the project aims, performed experimental and statistical analysis and drafted the manuscript. EA, SW, JC, WC, and JN contributed equally to experimental work. CC reviewed the manuscript and aided in the development of the concepts tested. All authors read and approved the final manuscript.

\section{Competing interests}

The authors declare that they have no competing interests.

Received: 6 December 2010 Accepted: 26 June 2011

Published: 26 June 2011

\section{References}

1. McLoughlin JM, Jensen EH, Malafa M: Resection of colorectal liver metastases: current perspectives. Cancer Control 2006, 13(1):32-41.

2. Neo JH, Ager El, Angus PW, Zhu J, Herath CB, Christophi C: Changes in the renin angiotensin system during the development of colorectal cancer liver metastases. BMC cancer 2010, 10:134.

3. Neo JH, Malcontenti-Wilson C, Muralidharan V, Christophi C: Effect of ACE inhibitors and angiotensin II receptor antagonists in a mouse model of colorectal cancer liver metastases. J Gastroenterol Hepatol 2007, 22(4):577-584.

4. Hunyady L, Catt KJ: Pleiotropic AT1 receptor signaling pathways mediating physiological and pathogenic actions of angiotensin II. Molecular endocrinology (Baltimore, Md 2006, 20(5):953-970.

5. Ager El, Chong WW, Wen SW, Christophi C: Targeting the antiogensin II type 2 receptor (AT2R) in colorectal liver metastases. Cancer cell international 2010, 10(1):19.

6. Stoll M, Steckelings UM, Paul M, Bottari SP, Metzger R, Unger T: The angiotensin AT2-receptor mediates inhibition of cell proliferation in coronary endothelial cells. The Journal of clinical investigation 1995, 95(2):651-657

7. Santos RA, Ferreira AJ: Angiotensin-(1-7) and the renin-angiotensin system. Current opinion in nephrology and hypertension 2007, 16(2):122-128.

8. Gallagher PE, Tallant EA: Inhibition of human lung cancer cell growth by angiotensin-(1-7). Carcinogenesis 2004, 25(11):2045-2052.

9. Menon J, Soto-Pantoja DR, Callahan MF, Cline JM, Ferrario CM, Tallant EA, Gallagher PE: Angiotensin-(1-7) inhibits growth of human lung adenocarcinoma xenografts in nude mice through a reduction in cyclooxygenase-2. Cancer research 2007, 67(6):2809-2815.

10. Petty WJ, Miller AA, McCoy TP, Gallagher PE, Tallant EA, Torti FM: Phase I and pharmacokinetic study of angiotensin-(1-7), an endogenous antiangiogenic hormone. Clin Cancer Res 2009, 15(23):7398-7404.

11. Uemura H, Hoshino K, Miyoshi Y, Kubota Y, Noguchi K, Takahashi S: Clinical trial and basic investigation: Angiotensin II receptor blocker decelerates PSA failure after radical retropubic prostatectomy. Journal of Clinical Oncology 2008, 26(15S):14676.

12. Kuruppu D, Christophi C, Bertram JF, O'Brien PE: Characterization of an animal model of hepatic metastasis. J Gastroenterol Hepatol 1996, 11(1):26-32.

13. Leenen $\mathrm{FH}$, Yuan B: Prevention of hypertension by irbesartan in Dahl S rats relates to central angiotensin II type 1 receptor blockade. Hypertension 2001, 37(3):981-984.

14. Zhang Y, Griendling KK, Dikalova A, Owens GK, Taylor WR: Vascular hypertrophy in angiotensin Il-induced hypertension is mediated by vascular smooth muscle cell-derived H2O2. Hypertension 2005, 46(4):732-737

15. Hirano T, Ran J, Adachi M: Opposing actions of angiotensin II type 1 and 2 receptors on plasma cholesterol levels in rats. Journal of hypertension 2006, 24(1):103-108.

16. Funao K, Matsuyama M, Kawahito Y, Sano H, Chargui J, Touraine JL, Nakatani T, Yoshimura R: Telmisartan is a potent target for prevention and treatment in human prostate cancer. Oncology reports 2008 , 20(2):295-300

17. Fujita M, Hayashi I, Yamashina S, Itoman M, Majima M: Blockade of angiotensin AT1a receptor signaling reduces tumour growth, angiogenesis, and metastasis. Biochemical and biophysical research communications 2002, 294(2):441-447.

18. Suganuma T, Ino K, Shibata K, Kajiyama H, Nagasaka T, Mizutani S, Kikkawa F: Functional expression of the angiotensin II type 1 receptor in human ovarian carcinoma cells and its blockade therapy resulting in suppression of tumour invasion, angiogenesis, and peritoneal dissemination. Clin Cancer Res 2005, 11(7):2686-2694.

19. Kosaka T, Miyajima A, Takayama E, Kikuchi E, Nakashima J, Ohigashi T, Asano T, Sakamoto M, Okita H, Murai M, Hayakawa M: Angiotensin II type 1 receptor antagonist as an angiogenic inhibitor in prostate cancer. Prostate 2007, 67(1):41-49.

20. Isobe A, Takeda T, Sakata M, Miyake A, Yamamoto T, Minekawa R, Nishimoto F, Oskamoto $Y$, Walker $C L$, Kimura T: Dual repressive effect of angiotensin II-type 1 receptor blocker telmisartan on angiotensin IIinduced and estradiol-induced uterine leiomyoma cell proliferation. Human reproduction (Oxford, England) 2008, 23(2):440-446.

21. Ishiguro $H$, Ishiguro $Y$, Kubota $Y$, Uemura $H$ : Regulation of prostate cancer cell growth and PSA expression by angiotensin II receptor blocker with peroxisome proliferator-activated receptor gamma ligand like action. Prostate 2007, 67(9):924-932.

22. Leung PS, Suen PM, Ip SP, Yip CK, Chen G, Lai PB: Expression and localization of AT1 receptors in hepatic Kupffer cells: its potential role in regulating a fibrogenic response. Regul Pept 2003, 116(1-3):61-69.

23. Allavena P, Sica A, Solinas G, Porta C, Mantovani A: The inflammatory micro-environment in tumour progression: The role of tumourassociated macrophages. Crit Rev Oncol Hematol 2007.

24. Ager El, Neo J, Christophi C: The renin-angiotensin system and malignancy. Carcinogenesis 2008, 29(9):1675-1684.

25. Deshayes F, Nahmias C: Angiotensin receptors: a new role in cancer? Trends Endocrinol Metab 2005, 16(7):293-299.

26. Fujiyama S, Matsubara H, Nozawa $Y$, Maruyama $K$, Mori $Y$, Tsutsumi $Y$, Masaki H, Uchiyama Y, Koyama Y, Nose A, Iba O, Tateishi E, Ogata N, Jyo N, Higashiyama S, Iwasaka T: Angiotensin AT(1) and AT(2) receptors differentially regulate angiopoietin-2 and vascular endothelial growth factor expression and angiogenesis by modulating heparin bindingepidermal growth factor (EGF)-mediated EGF receptor transactivation. Circulation research 2001, 88(1):22-29.

27. Silvestre JS, Tamarat R, Senbonmatsu T, Icchiki T, Ebrahimian T, Iglarz M, Besnard S, Duriez M, Inagami T, Levy Bl: Antiangiogenic effect of angiotensin II type 2 receptor in ischemia-induced angiogenesis in mice hindlimb. Circulation research 2002, 90(10):1072-1079.

28. Clere N, Corre I, Faure S, Guihot AL, Vessieres E, Chalopin M, Morel A, Coqueret $\mathrm{O}$, Hein L, Delneste Y, Paris F, Henrion D: Deficiency or blockade of angiotensin II type 2 receptor delays tumourigenesis by inhibiting malignant cell proliferation and angiogenesis. International journal of cancer 2010, 127(1):2279-2291.

29. Pawlikowski M: Immunohistochemical detection of angiotensin receptors AT1 and AT2 in normal rat pituitary gland, estrogen-induced rat pituitary tumour and human pituitary adenomas. Folia histochemica et cytobiologica / Polish Academy of Sciences, Polish Histochemical and Cytochemical Society 2006, 44(3):173-177.

30. Ptasinska-Wnuk D, Lawnicka H, Fryczak J, Kunert-Radek J, Pawlikowski M: Angiotensin peptides regulate angiogenic activity in rat anterior pituitary tumour cell cultures. Endokrynologia Polska 2007, 58(6):478-486.

31. Yoshiji H, Kuriyama S, Fukui H: Perindopril: possible use in cancer therapy. Anti-cancer drugs 2002, 13(3):221-228.

32. Wang L, Cai SR, Zhang CH, He YL, Zhan WH, Wu H, Peng JJ: Effects of angiotensin-converting enzyme inhibitors and angiotensin II type 1 receptor blockers on lymphangiogenesis of gastric cancer in a nude mouse model. Chinese medical journal 2008, 121(21):2167-2171.

33. Nakagawa T, Kubota T, Kabuto M, Kodera T: Captopril inhibits glioma cell invasion in vitro: involvement of matrix metalloproteinases. Anticancer research 1995, 15(5B):1985-1989.

\section{Pre-publication history}

The pre-publication history for this paper can be accessed here: http://www.biomedcentral.com/1471-2407/11/274/prepub

doi:10.1186/1471-2407-11-274

Cite this article as: Ager et al:: Altered efficacy of AT1R-targeted treatment after spontaneous cancer cell-AT1R upregulation. BMC Cancer $201111: 274$ 